\title{
Wide Field-of-View Flexible Wavelength Routing and Multicasting for Multi-user Bi-directional Indoor Optical Wireless Communications
}

\author{
Feng Feng ${ }^{1 *}$, Paramin Sangwongngam ${ }^{2}$, Grahame Faulkner ${ }^{2}$, and Dominic O'Brien ${ }^{2}$ \\ ${ }^{1}$ School of Precision Instruments and Opto-electronics Engineering, Tianjin University, Tianjin 300072, China \\ ${ }^{2}$ Department of Engineering Science, University of Oxford, Parks Road,OX1 3PJ, Oxford UK \\ *feng_feng@tju.edu.cn
}

\begin{abstract}
Between an SLM-based base station with $\pm 30^{\circ}$ FOV and 4 steering-mirrorbased nomadic terminals with $\pm 50^{\circ} \mathrm{FOV}$, we demonstrate flexible wavelength routing and multicasting with bi-directional optical wireless transmissions at $25 \mathrm{~Gb} / \mathrm{s}$ over 4 metres.

(C) 2020 The Author(s)
\end{abstract}

OCIS codes: $060.0060,070.0070$.

\section{Introduction}

Next-generation passive optical network (PON) technologies enable fiber-to-the-home/building (FTTH/B) networks that deliver high capacity (10 Gb/s and beyond) Internet service to end-users. As an attractive solution, OWC using beam-steered infrared narrow beams directly from the optical fiber networks can effectively provide high capacity wireless access [1]. Due to the narrow field-of-view (FOV) of single mode fibre based components, beam steering at both the base station and user terminals is required by such OWC systems to offer wide FOV. Additionally, tracking and localization are important for such systems to be practical, as demonstrated in [2].

In the second next-generation PON (NG-PON2), time-division and wavelength-division multiplexing (TWDM) have been proposed as the primary solution [3]. As a result, further from optical broadcasting only [4], flexible wavelength routing and multicasting to different nomadic user terminals are highly desired by OWC systems using beam-steered infrared narrow beams from optical fibres carrying TWDM signals. In this paper, we experimentally demonstrate wide FOV flexible wavelength routing and multicasting in a bi-directional indoor OWC system over a range of 4 metres, between a spatial light modulator (SLM) based beam steering base station with $\pm 30^{\circ}$ FOV and four (1-to-2 multicasting of 2 wavelength channels) nomadic user terminals that employ mirror-based steering with $\pm 50^{\circ} \mathrm{FOV}$. In the downstream direction, the SLM-based base station routes multiplexed wavelengths from an optical fibre to desired terminals using separate areas on the SLM. For multicasting, the base station multicasts one particular multiplexed wavelength from the optical fibre to multiple desired user terminals. In the upstream direction, light from an optical fibre at a different wavelength propagates along with the same free-space optical path from a user terminal to the base station. Constructed composite phase masks on separate areas of the SLM at the base station steer downstream optical transmissions at different wavelengths and corresponding upstream optical transmissions at the same time. PAM-4 modulation is used to transmit $25 \mathrm{~Gb} / \mathrm{s}$ in both upstream and downstream data transmissions. BER below the FEC threshold is achieved for the data transmissions.
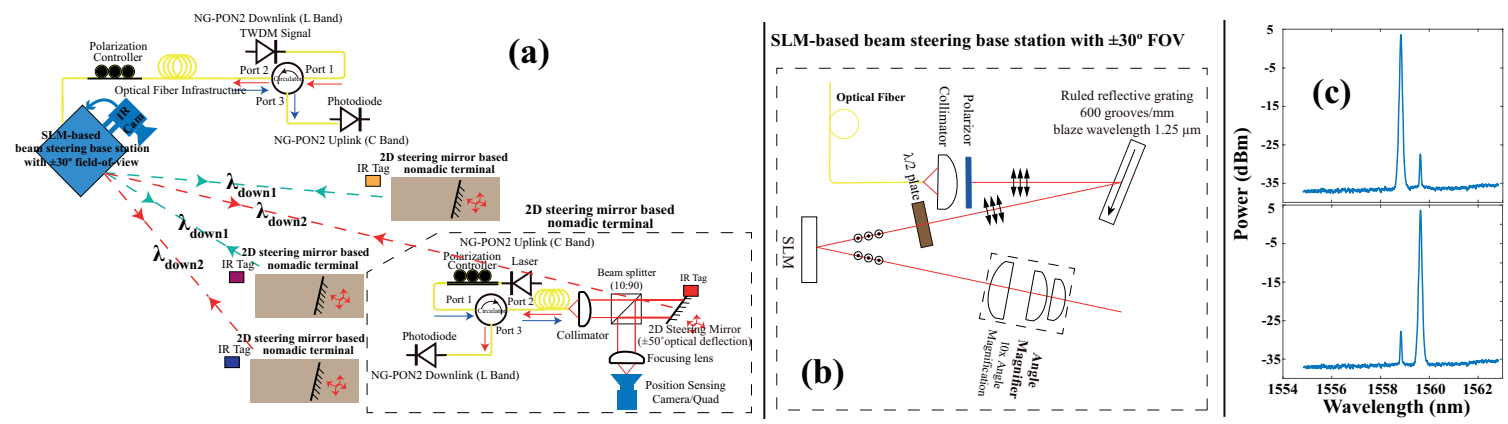

Fig. 1. (a) System design of the wide FOV indoor bi-directional OWC system to integrate with FTTH/B networks enabled by TWDM NG-PON2 technologies. (b) Design of the SLM-based beam steering base station that has $\pm 30^{\circ} \mathrm{FOV}$ and performs flexible wavelength routing and multicasting. (c) OSA measurements at the receiving end of two user terminals (wavelength routing). 


\section{System design of the indoor bi-directional OWC system}

The indoor OWC system is designed to not only route a particular wavelength to an assigned user terminal but also multicast data-carrying time frames at an operating wavelength to multiple desired user terminals, integrating with FTTH/B networks enabled by TWDM NG-PON2 technologies. NG-PON2 technologies specify the upstream and downstream wavelength bands located respectively in the C-band and L-band. Multiple wavelengths can be routed and multicast at the same time and the routing and multicasting are reconfigurable. Fig. 1 (a) illustrates the system design. The flexible wavelength routing and multicasting are enabled by the SLM-based beam steering base station. The SLM-based base station has $\pm 30^{\circ} \mathrm{FOV}$, which is achieved by $10 \times$ angle magnification of the SLM steering range $\left( \pm 3^{\circ}\right)$ using a telescope lens system. Fig. 1 (b) shows the design of the SLM-based beam steering base station. A ruled reflective grating $(600$ grooves $/ \mathrm{mm}, 1250 \mathrm{~nm}$ blaze wavelength $)$ is mounted in the Littrow configuration. It spatially disperses the downstream optical transmissions that consist of $N$ WDM channels onto an SLM, such that each wavelength channel is imaged onto a separate area on the SLM along the dispersion direction. For a downstream wavelength channel using a separate area on the SLM, a composite phase mask is constructed by superposition weighted beam steering phase masks for both downstream and upstream transmissions of all associated user terminals, in the same way as described in [4]. The steering mirrors used by nomadic user terminals have up to $\pm 50^{\circ}$ optical deflection. The tracking and localization scheme proposed in our point-to-multipoint indoor OWC system detailed in [4] is adopted here, as shown in Fig. 1 (a). Polarization controls at the base station and user terminals align the polarization of both the downstream and upstream optical transmissions to the required states for high diffraction efficiency.

\section{Experimental results and conclusion}

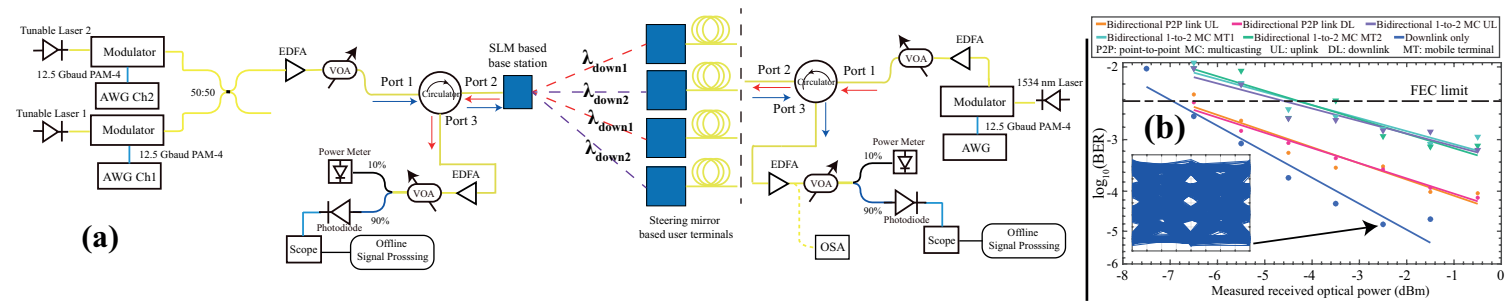

Fig. 2. (a) Experimental system. (b) BER curves versus received optical power at 25 Gb/s PAM-4.

Fig. 2 (a) shows the experimental system for data transmission tests. Due to the lack of L-band EDFAs, we selected $1534 \mathrm{~nm}$ as upstream wavelength and a downstream wavelength range from $1554.13 \mathrm{~nm}$ to $1561.42 \mathrm{~nm}$. The SLM-based base station supports wavelength routing and multicasting for up to $10 \mathrm{ITU} 100 \mathrm{GHz}$-spaced wavelengths. Two tunable lasers operating at adjacent wavelengths are independently modulated at $25 \mathrm{~Gb} / \mathrm{s}$ PAM4. By tuning the wavelengths of the two tunable lasers, crosstalk between all supported adjacent wavelength channels is measured below $-30 \mathrm{~dB}$. Fig.1 (c) shows typical optical spectrum measurements at two nomadic terminals to which two adjacent wavelength channels at $1558.98 \mathrm{~nm}$ and $1559.79 \mathrm{~nm}$ are respectively routed. The transmit output optical power of each of two downstream wavelength channels and each upstream channel is set to $4 \mathrm{dBm}$ to make the OWC system eye safe. The measured point-to-point (wavelength routing only) optical link loss is $21.2 \mathrm{~dB}$. Both the bi-directional operation $(3 \mathrm{~dB})$ between the base station and a user terminal and 1-to- 2 wavelength multicasting $(3 \mathrm{~dB})$ introduce an extra $6 \mathrm{~dB}$ link loss in total. Fig. 2 (b) shows the BER measurements. Compared with the performance of a single downlink, the bi-directional operation costs $\sim 0.5 \mathrm{~dB}$ power penalty at the FEC threshold. Further including 1-to-2 multicasting results in $\sim 2.6 \mathrm{~dB}$ power penalty at the FEC threshold. To conclude, we have demonstrated wide FOV flexible wavelength routing and multicasting in an indoor bidirectional OWC system. This work is supported by UK EPSRC project COALESCE (EP/PO03990/1).

\section{References}

1. T. Koonen, "Indoor optical wireless systems: Technology, trends, and applications," J. Light. Technol. 36, 1459-1467 (2018).

2. A. Gomez and et al., "A $50 \mathrm{gb} / \mathrm{s}$ transparent indoor optical wireless communications link with an integrated localization and tracking system," J. Light. Technol. 34, 2510-2517 (2016).

3. D. Nesset, "Ng-pon2 technology and standards," J. Light. Technol. 33, 1136-1143 (2015).

4. F. Feng and et al., "Wide field-of-view optical broadcasting for bi-directional indoor optical wireless communications employing pam-4 modulation," Opt. Lett. 44, 6009-6012 (2019). 